\title{
Testes Paramétricos e Não-Paramétricos de Reversão para a Média da Rendibilidade de Índices do Mercado Accionista
}

\author{
Nelson Manuel de P. B. da Costa Areal \\ Manuel José da Rocha Armada
}

\section{ResUMO}

Recentemente vários estudos empíricos têm questionado a hipótese de a rendibilidade das acções seguir um percurso aleatório, apresentando um comportamento de reversão/aversão para a média. O presente trabalho de investigação utiliza rendibilidades nominais, reais e em excesso a uma rendibilidade isenta de risco, de vários índices (na sua maioria, criados especificamente para a realização deste estudo), calculadas para vários horizontes temporais de médio e longo prazo, sendo um estudo pioneiro no mercado português de capitais. Foi utilizada a metodologia da regressão pelo método dos mínimos quadrados. A significância estatística foi aferida pela utilização de testes de significância estatística paramétricos e não-paramétricos. A utilização de testes não-paramétricos dispensa a verificação da hipótese de normalidade, sendo eficiente mesmo na presença de autocorrelação e heteroscedasticidade não condicional, apresentando ainda a vantagem de não ser necessário confiar em correcções que apenas têm validade assimptótica, o que constitui grande vantagem devido ao limitado número de observações destes estudos. Detectamos a existência de comportamentos de reversão para a média e de aversão à média das rendibilidades das acções, resultados estes que não colocam, necessariamente, em causa a hipótese de eficiência do mercado accionista.

Palavras-chaves: eficiência dos mercados; anomalias nos mercados de capitais; métodos de simulação estatística; métodos de Monte Carlo.

\begin{abstract}
In the last few years several research studies challenged the traditional weak form efficiency tests of the stock market. These studies suggested mean aversion/reversion behaviour of stock market returns. This is a pioneer study of the Portuguese stock market, and uses nominal, real and excess returns, computed for various longer-horizons. The ordinary least square regression methodology was used. The statistical significance of the results was studied using traditional parametric tests as well as non-parametric tests. The non-parametric test does not require the assumption of normality and the use of correction procedures only valid asymptotically, which an advantage is due to the relative small sample size of these studies. The evidence is mixed, as we detected the presence of tendencies towards mean aversion and mean reversion. These results, however, do not necessarily reject the weak form market efficiency hypothesis.
\end{abstract}

Key words: market efficiency; stock market anomalies; statistical simulation methods; Monte Carlo methods. 


\section{INTRODUÇÃO}

Um mercado eficiente é aquele que incorpora de forma completa e rápida toda a informação que está disponível (Fama, 1970, 1976). A hipótese da forma fraca de eficiência do mercado de capitais, também designada por alguns autores de hipótese de percursos aleatórios, prediz que não é possível obter-se uma rendibilidade (ajustada ao risco) superior à do mercado pela utilização de informações contidas na sucessão cronológica histórica dos preços dos activos financeiros. Sendo assim, se os mercados de capitais forem eficientes na sua forma fraca, não haverá vantagem em utilizar qualquer estratégia de comercialização dos activos financeiros, que tenha por base informações do passado, uma vez que estas estratégias não conseguiriam ultrapassar, sistematicamente, uma simples estratégia de aquisição e manutenção desses activos.

Em 1970, Fama conclui que os testes sobre a eficiência do mercado de capitais na sua forma fraca são numerosos, e os resultados são consistentes com essa hipótese. "Em resumo, a evidência em suporte do modelo de eficiência do mercado de capitais é extensa e (de uma forma, um tanto única em economia) a evidência contra esse modelo é escassa" (Fama 1970, p. 416).

Desde então, surgiram inúmeros estudos sobre a hipótese dos mercados de capitais seguirem um percurso aleatório, tendo-se detectado várias anomalias ${ }^{(1)}$. Fama em 1991 já afirma que os novos estudos sobre a eficiência do mercado accionista revelam que é possível prever as rendibilidades futuras através de rendibilidades do passado e de várias outras variáveis. "Os novos testes rejeitam assim, o antigo modelo de eficiência de mercado com rendibilidade esperada constante, que parecia adequado aos estudos anteriores" (Fama, 1991, p. 1577).

Uma das últimas anomalias detectadas diz respeito à existência de padrões de comportamento das rendibilidades de médio e longo prazo de índices de acções. Esta evidência sugere que os índices de acções possuem uma tendência para a reversão para a média, quando as rendibilidades são medidas com base em horizontes de investimento de médio e longo prazo. Esta anomalia foi inicialmente detectada por Fama e French (1988) e mais tarde rejeitada, entre outros, por Kim, Nelson e Startz (1991), McQueen (1992) e Richardson (1993a).

Este artigo investiga pela primeira vez, o comportamento das rendibilidades para horizontes de investimento de médio e longo prazo de índices de acções do mercado português. A grande maioria da investigação sobre o tema tem incidido sobre mercados desenvolvidos, especialmente sobre os mercados norte-america- 
nos, que são em muitos aspectos bastante diferentes do mercado accionista português. Este mercado caracteriza-se por um número relativamente reduzido de emissões admitidas à cotação, capitalização bolsista e volume de transacções, quando comparado com os mercados mais desenvolvidos.

Como já foi demonstrado por outros estudos, o valor da capitalização bolsista da empresa e o sector de actividade em que se insere podem captar comportamentos diferentes das suas rendibilidades (King, 1966; Banz, 1981). Desta forma, foram objecto de estudo tanto índices ponderados como não ponderados, bem como índices de rendibilidades de carteiras criadas com base no sector de actividade das empresas e com base no valor da sua capitalização bolsista.

Uma vez que a evidência de reversão para a média e de aversão à média pode ser sensível ao tipo de testes de significância estatística utilizado, foram realizados testes de significância paramétricos e não-paramétricos.

\section{A Reversão para a Média da Rendibilidade de Médio e Longo Prazo DAS ACÇÓES}

Os estudos de reversão para a média foram inspirados no artigo de Summers (1986), em que o autor apresenta um modelo de comportamento das rendibilidades alternativo ao modelo de percursos aleatórios. Summers (1986) demonstra que se o preço das acções segue modelo que contenha um componente estacionário que lentamente diminua a sua influência no comportamento desses preços, então os testes tradicionais da forma fraca de eficiência do mercado de capitais iriam apenas ser eficazes se aplicados a sucessões cronológicas de dimensão muito superior àquelas de que dispomos actualmente.

Summers (1986) demonstrou que os testes habitualmente utilizados para verificar se as séries temporais seguiam um percurso aleatório não seriam apropriados, havendo situações em que, apesar de o preço dos activos estar afastado do seu valor de equilíbrio em cerca de $30 \%$, devido à componente estacionária dos preços, os testes de eficiência do mercado na sua forma fraca não conseguiam rejeitar a hipótese nula de que os preços seguiam um percurso aleatório. Os resultados obtidos sugerem que seria necessária uma série cronológica com observações respeitantes a cerca de 5.000 anos, por forma a haver uma probabilidade de 50\% de um teste tradicional de percurso aleatório rejeitar a hipótese nula, quando ela é falsa.

Seguindo este raciocínio, Fama e French (1988) apresentam um modelo de formação de preços que combina um percurso aleatório com movimentos estacioná- 
rios da rendibilidade dos activos. O modelo sugerido por Fama e French (1988) adequa-se à classe de modelos mais genérica em que parte da alteração esperada da rendibilidade de cada mês é permanente (rendibilidade esperada) e a restante parte é gradualmente eliminada, justificando, assim, a existência de componentes temporárias e permanentes no preço das acções. A existência dessa componente temporária implica que as rendibilidades dos activos irão apresentar uma autocorrelação negativa, sendo conseqüentemente, pelo menos em parte, previsíveis $^{(2)}$.

O resultado do desenvolvimento analítico do modelo permite-nos concluir que, mesmo que o valor de uma acção contenha desvios estacionários temporários, ao longo de intervalos reduzidos irá apresentar uma forma de dependência cronológica reduzida ou nula. No entanto, quando consideramos períodos mais longos de tempo, essa dependência cronológica será revelada sob a forma de reversão para a média.

\section{Evidências Empíricas}

Recentemente vários estudos empíricos ${ }^{(3)}$, em que foram utilizados rendibilidades calculadas para horizontes de investimento de médio e longo prazo, apresentam uma autocorrelação negativa. São disso exemplo os resultados de Fama e French (1988) e Poterba e Summers (1988), que estudam a existência do fenómeno no mercado accionista dos Estados Unidos da América e noutros mercados internacionais. Fama e French (1988) constatam que o fenómeno diminui de intensidade a partir dos anos quarenta. Richardson e Stock (1989) apresentam uma reinterpretação dos resultados obtidos por Fama e French (1988) e por Poterba e Summers (1988), o que os leva a concluir que a evidência de reversão para a média é muito menos significativa do que anteriormente tinha sido reportado.

Jegadeesh (1990), utilizando uma metodologia ligeiramente diferente que a utilizada pelos autores anteriormente citados, conclui que o fenómeno da reversão para a média está inteiramente concentrado no mês de janeiro, e que este fenómeno pode ser detectado no período posterior ao da Segunda Guerra Mundial.

Kim, Nelson e Startz (1991) demonstram que as evidências de reversão para a média poderão ser incorrectas devido à não normalidade das rendibilidades das acções, concluindo só ser possível detectar reversão para a média no período anterior à Segunda Guerra Mundial. No período posterior à Segunda Guerra Mundial os autores constatam a existência de uma tendência para a persistência, a que designam de aversão à média.

McQueen e Thorley (1991) utilizam a metodologia das cadeias de Markov, que permite a consideração de um comportamento não linear da rendibilidade de mé- 
dio e longo prazo das acções. Estes testes indicam um comportamento de reversão para a média da rendibilidade, mesmo para o período posterior à Segunda Guerra Mundial.

McQueen (1992) incorpora nos seus testes vários dos avanços metodológicos que surgiram desde os primeiros estudos sobre o tema. Os seus resultados não conseguem rejeitar a hipótese de que o mercado accionista segue um percurso aleatório, com excepção das rendibilidades do período de 1926 a 1946, não existindo nenhum outro período em que isso aconteça. Mesmo quando é realizado um teste conjunto, considerando os 10 horizontes temporais simultaneamente, no período de 1926 a 1946, não é possível rejeitar a hipótese de que a rendibilidade segue percurso aleatório.

Richardson (1993b) demonstra que a interpretação individual dos resultados das medidas de reversão para a média de vários horizontes temporais poderá falsear os resultados, sugerindo um teste conjunto de significância para as medidas de todos os horizontes temporais.

\section{Descrição da BASE de DAdos}

Os estudos sobre reversão para a média das rendibilidades dos activos necessitam de um grande número de observações para a sua realização. Este facto não constitui grande obstáculo no mercado norte-americano, onde se consegue, com relativa facilidade, obter sucessões cronológicas de índices do mercado accionista desde 1871 até ao presente.

Em Portugal tal não se verifica. Presentemente, existem vários índices gerais de acções, como sejam: o BVL - Geral, o BVL-30, o PSI-20 e o BTA, para além de índices sectoriais. No entanto só muito recentemente teve início o cálculo destes índices. O BVL - Geral começou a ser calculado em 1988, os índices sectoriais da Bolsa de Valores de Lisboa (BVL) tiveram início no ano de 1991, o PSI-20 e BVL-30 no ano de $1993^{(4)}$. O índice mais antigo do mercado português de capitais é do Banco Totta \& Açores (BTA) que teve início em 1977. O índice PSI-20 é da responsabilidade da Associação da Bolsa de Valores do Porto (BDP), mas como a generalidade dos índices que são transaccionados em mercados de produtos financeiros derivados, não é ajustado ao pagamento de dividendos.

Como, em primeiro lugar, os índices de acções que actualmente existem não consideram um período de tempo suficientemente grande para um estudo sério do fenómeno da reversão para a média, com excepção do índice BTA e, em segundo lugar, como não existem no mercado accionista português índices não ponderados 
e índices calculados para carteiras criadas de acordo com a dimensão relativa da sua capitalização bolsista, surgiu a necessidade de criar índices para a realização deste estudo.

A metodologia utilizada para a criação dos índices de acções seguiu de perto a metodologia sugerida por Costa e Sousa (1988), por Fonseca, Confraria e Pinheiro (1990), e pela Associação da Bolsa de Valores do Porto (1995).

Os índices foram calculados diariamente, durante o período de 5 ou 6 de janeiro de 1983 a 23 de dezembro de $1996^{(5)}$. A escolha deste período é justificada pela inexistência, em suporte informático, de dados sobre o mercado anteriores a 1983, o que inviabilizou a recolha e tratamento manual da série cronológica de preços das emissões cotadas em mercado oficial. Se a este facto adicionarmos a reduzida liquidez que o mercado oficial da BVL possuiu entre 1977 e 1980, não nos pareceu justificável a recolha manual dessa informação. Tanto mais que as publicações anteriores a 1983 nos boletins de cotação da BVL não nos forneciam todos os dados necessários para o cálculo do índice, nomeadamente as condições e características das alterações de capital das sociedades.

Idealmente, teríamos preferido incluir as 343 emissões que foram admitidas a cotação no mercado oficial e posteriormente no mercado de cotações oficiais da BVL entre 5 de janeiro de 1983 e 23 de dezembro de 1996; no entanto a morosidade do processo de recolha e tratamento dos dados e a reduzida liquidez de muitas dessas emissões condicionaram a selecção das emissões que fazem parte dos índices criados.

Os critérios estabelecidos para a selecção da amostra tiveram como objectivo permitir, em primeiro lugar, criar um índice geral (ponderado e não ponderado) que fosse representativo, em termos de capitalização bolsista, do mercado de cotações oficiais da BVL. Em segundo lugar, estabelecemos um critério mínimo para inclusão da emissão na carteira, de acordo com a sua liquidez: todas as emissões que compõem a carteira do índice deveriam ter sido transaccionadas em pelo menos $60 \%$ dos dias em que era possível transaccioná-las. Por último, seleccionamos as emissões de tal forma que fosse possível a criação de índices sectoriais e por quartis da capitalização bolsista, tarefa que não foi facilitada pela elevada concentração da capitalização bolsista e de liquidez, num número reduzido de emissões.

Após a recolha de dados relativos a 153 emissões, e da análise das séries cronológicas de preços, optamos por reduzir o número de emissões a incluir na carteira do índice para 109, uma vez que várias dessas acções não cumpriam com o nosso critério de liquidez. Viu-se, assim, reduzida a representatividade em termos de capitalização bolsista em relação ao mercado, mas obteve-se um ganho em rela- 
ção ao objectivo de representatividade das expectativas do investidor médio ${ }^{(6)}$.

O índice BTA foi obtido no Departamento de Pesquisa da sociedade financeira de corretagem TottaDealer S. A. Os dados obtidos compreendem os valores diários do índice BTA entre 1 de abril de 1977 e 12 de dezembro de 1996.

Os valores do índice geral de preços do consumidor (continente) foram obtidos no Centro de Estatísticas Gerais do Departamento de Estatísticas e Estudos Económicos (DEE) do Banco de Portugal.

Para o cálculo das rendibilidades em excesso a uma taxa isenta de risco foi necessário obter informações sobre a rendibilidade de algum investimento de curto prazo, cujo risco fosse inexistente ou mínimo. O investimento escolhido que se adequa a essas condições foi o investimento em bilhetes do Tesouro a 91 dias. As taxas médias ponderadas de colocação no mercado primário dos Bilhetes de Tesouro a 91 dias para cada emissão, desde a criação deste instrumento financeiro, foram fornecidas pelo Núcleo de Mercados Interbancários do Departamento de Operações de Crédito e Mercados (DOCM) do Banco de Portugal.

A primeira emissão de Bilhetes de Tesouro ocorreu em 14 de agosto de 1985, não existindo, de acordo com o DOCM, para datas anteriores nenhum instrumento de dívida pública equiparável. Desta forma, não será possível estudar o comportamento das rendibilidades em excesso para todo o período em análise, estando a análise destas rendibilidades naturalmente condicionada pela falta de dados.

Pretende-se com o cálculo das rendibilidades em excesso a uma rendibilidade isenta de risco a comparação de uma estratégia de aquisição e manutenção do índice em relação a um investimento de curto prazo, isento ou com risco reduzido; assim a rendibilidade isenta de risco para cada mês foi obtida pela última emissão de Bilhetes de Tesouro a 91 dias do mês anterior ${ }^{(7)}$.

\section{Metodologia Utilizada}

Não existe, de que tenhamos conhecimento, nenhum outro estudo que tenha investigado o comportamento das rendibilidades de médio e longo prazo do mercado accionista português, pelo que este é um estudo pioneiro neste mercado. Sendo assim, interessa-nos não só investigar o comportamento das rendibilidades dos índices de acções, como a consistência dos resultados obtidos através de várias metodologias de teste de significância estatística desses resultados.

Para verificar a significância estatística do teste de hipótese podem ser utiliza- 
dos testes paramétricos ou não-paramétricos. Os testes paramétricos assumem sempre determinada distribuição de probabilidade para as variáveis em análise. Por exemplo, quando se testa a significância estatística de um coeficiente da regressão pelo método dos mínimos quadrados, é necessário assumir que os termos de perturbação aleatória são normalmente distribuídos. Infelizmente, a realidade nem sempre satisfaz esse pressuposto, transformando esses coeficientes em estimadores não eficientes. Este problema é tanto mais significativo quanto mais reduzida for a dimensão da amostra com que estamos a trabalhar. A utilização de testes não-paramétricos permite-nos testar a significância estatística dos resultados, dispensando qualquer pressuposto no que concerne à distribuição de probabilidade das variáveis.

Estudos anteriores sugerem que as evidências podem estar incorrectamente interpretadas, devido ao pressuposto de normalidade necessário para utilizar os testes de significância paramétricos (Richardson e Stock, 1989; Kim, Nelson e Startz, 1991; McQueen, 1992). Para permitir uma comparação entre os resultados obtidos pelo presente estudo e os resultados obtidos em estudos anteriores, foram utilizados testes de significância estatística paramétricos e não-paramétricos para os coeficientes da regressão.

Foram analisadas as rendibilidades nominais, reais e em excesso para três índices gerais, quatro índices criados com base na dimensão relativa da capitalização bolsista das empresas, e quatro índices criados com base no sector de actividade económica das empresas.

\section{A Regressão pelo Método dos Mínimos Quadrados}

Esta metodologia é inspirada no modelo de Fama e French (1988), através do qual os autores concluem: se os preços das acções seguem um modelo de comportamento com componentes temporárias e permanentes, então a regressão das rendibilidades de médio e longo prazo, em períodos desfasados no tempo, irá revelar uma tendência de reversão para a média. Para se aplicar a metodologia é necessário utilizar a seguinte regressão:

$$
r_{(t, t+k)}=\alpha+\beta_{k} r_{(t-k, t)}+\varepsilon_{t}
$$

Em que $\mathrm{r}_{(\mathrm{t}, \mathrm{t}+\mathrm{k})}$ é a rendibilidade calculada com base em rendibilidades mensais para o período de $t$ a $t+k$ em que $\mathrm{k}=12,24,36, \ldots$ meses.

O beta da regressão $\left(\beta_{\mathrm{k}}\right)$ representa a autocorrelação de primeira ordem das rendibilidades de k períodos. Um $\beta$ negativo indicará a presença de autocorrelação 
negativa das rendibilidades dos índices de acções, ou seja de reversão para a média. Um coeficiente $\beta$ igual a zero indicará que as rendibilidades seguem percurso aleatório, e um $\beta$ maior que zero poderá indicar a presença de aversão à média das rendibilidades dos índices de acções.

O período de que dispomos apenas nos proporciona um número relativamente reduzido de observações. Por exemplo, se trabalhássemos com séries de rendibilidades de acções calculadas com base em intervalos de tempo de 5 anos, nem conseguiríamos obter 3 observações, o que seria claramente insuficiente para realizar qualquer teste estatístico. Para contornar este problema, seguiu-se a forma de cálculo de que se vale a generalidade dos autores, incluindo Fama e French (1988), utilizando-se observações mensais justapostas, o que nos permitiu aumentar significativamente o número de observações.

A escolha desta forma de cálculo das rendibilidades tem como consequência a criação de um enviesamento dos resultados, uma vez que induz um efeito de autocorrelação negativo, que aumenta à medida que aumenta o período de tempo em que as rendibilidades são calculadas. Esse enviesamento irá impossibilitar a interpretação correcta dos resultados, pelo que será necessário retirar-se o enviesamento provocado pela forma de cálculo das rendibilidades anuais.

Para além do enviesamento provocado pela utilização de observações justapostas, Marriot e Pope (1954) e Kendall (1954) demonstram que, em amostras de reduzida dimensão, as estimativas dos coeficientes da regressão são enviesadas.

Para remover esse enviesamento, estimou-se o grau de enviesamento em cada coeficiente da regressão, por meio de simulação, tal como Fama e French (1988). As rendibilidades mensais foram simuladas pela geração de sucessões cronológicas de números independentes, seguindo uma distribuição normal $(\mathrm{N} \sim(0,1))$. Essas rendibilidades mensais foram então somadas para obter o valor das rendibilidades anuais, obtendo-se, assim, observações justapostas, para vários horizontes de investimento ( $\mathrm{k}=12,24,36, \ldots$ meses). Finalmente, calcularam-se os coeficientes da regressão para as rendibilidades simuladas.

Após 25.000 iterações obtiveram-se os valores esperados dos coeficientes beta da regressão, para o caso em que as rendibilidades são independentes; esses valores são dados pela Tabela 1. Pela análise dessa tabela podemos observar que a utilização de observações justapostas para o cálculo das rendibilidades anuais provoca um efeito de autocorrelação negativa, que nos poderá induzir em erro, se não corrigirmos os $\beta$ estimados desse enviesamento. 


\section{Tabela 1: Valor Esperado do Beta da Regressão Dado pela Expressão [4] quando Aplicada a Sucessões Cronológicas de Reduzida Dimensão que Fazem Uso de Observações Justapostas para o Cálculo de Rendibilidades de Médio e Longo Prazo}

\begin{tabular}{|c|c|c|c|c|c|c|c|c|c|}
\hline \multirow[b]{2}{*}{ - Cronológica } & \multicolumn{9}{|c|}{ Períodos } \\
\hline & 1 & 2 & 3 & 4 & 5 & 6 & 7 & 8 & 9 \\
\hline 133 Observações & -0.13828 & -0.30571 & -0.46269 & -0.50111 & -0.50458 & & & & \\
\hline 164 Observações & -0.11130 & -0.24166 & -0.38349 & -0.49603 & -0.50922 & -0.50446 & & & \\
\hline 166 Observações & -0.10866 & -0.23874 & -0.37885 & -0.48682 & -0.49724 & -0.50105 & & & \\
\hline 235 Observações & -0.07428 & -0.15846 & -0.25107 & -0.35431 & -0.44345 & -0.49434 & -0.49837 & -0.50533 & -0.49674 \\
\hline
\end{tabular}

Para testarmos a significância estatística de qualquer estimador dos coeficientes da regressão, é possível utilizar testes de significância paramétricos, como, por exemplo, o teste de $t$, e testes não-paramétricos. Para aplicarmos os testes paramétricos necessitamos não só de estimadores dos coeficientes da regressão sem enviesamento, como também estimadores eficientes. Com base nos valores esperados obtidos para enviesamento, é possível expurgar os estimadores do enviesamento provocado pela utilização de observações justapostas e de um número relativamente reduzido de observações. Para assegurar que os estimadores são eficientes é necessário que os termos de perturbação aleatória (resíduos) da regressão sigam uma distribuição normal, sejam independentes e homocedásticos.

Todos os pressupostos acima referidos foram testados formalmente. Para testar a hipótese de normalidade dos resíduos foram utilizados os testes de adequação de Qui-Quadrado, Kolmogorov-Smirnov, Anderson-Darling e Jarque-Bera. Para a hipótese de homocedasticidade foi utilizado o teste de White (1980) e para a hipótese de autocorrelação dos resíduos da regressão utilizou-se um teste de multiplicador de Lagrange.

A Tabela 2 apresenta um resumo dos testes de normalidade dos resíduos das regressões efectuados para todos os horizontes de investimento e para todos os índices de rendibilidade considerados (nominais, reais e em excesso) ${ }^{(8)}$. Pela análise destes resultados é possível afirmar que, para a vasta maioria dos casos, é possível rejeitar a hipótese de que os resíduos da regressão sigam uma distribuição normal, utilizando mais do que um teste de adequação.

É também possível rejeitar a hipótese de homocedasticidade e/ou de independência dos resíduos da regressão, para a grande maioria dos índices, rendibilidades e horizontes de investimento considerados, como é possível observar pelo resumo dos resultados desses testes proporcionado pela Tabela 2 . 
Tabela 2: Resumo dos Resultados dos Testes de Adequação, Heterocedasticidade e Autocorrelação, Efectuados aos Resíduos da Regressão pelo Método dos Mínimos Quadrados

\begin{tabular}{|c|c|c|c|c|c|c|c|c|c|}
\hline \multirow[b]{2}{*}{ Índices } & \multicolumn{9}{|c|}{ Horizonte de investimento (em anos) } \\
\hline & 1 & 2 & 3 & 4 & 5 & 6 & 7 & 8 & 9 \\
\hline \multicolumn{10}{|l|}{ BTA } \\
\hline Rend. Nominais & $\mathrm{CS}, \mathrm{KS}, \mathrm{AD}, \mathrm{JB}, \mathrm{H}, \mathrm{A}$ & $\mathrm{CS}, \mathrm{KS}, \mathrm{AD}, \mathrm{JB}, \mathrm{H}, \mathrm{A}$ & $\mathrm{CS}, \mathrm{KS}, \mathrm{AD}, \mathrm{JB}, \mathrm{H}, \mathrm{A} C \mathrm{C}$ & $\mathrm{CS}, \mathrm{KS}, \mathrm{AD}, \mathrm{JB}, \mathrm{H}, \mathrm{A}$ & $\mathrm{CS}, \mathrm{KS}, \mathrm{AD}, \mathrm{JB}, \mathrm{H}, \mathrm{A}$ & $\mathrm{CS}, \mathrm{KS}, \mathrm{AD}, \mathrm{JB}, \mathrm{H}, \mathrm{A}$ & $\mathrm{H}, \mathrm{A}$ & $\mathrm{CS}, \mathrm{KS}, \mathrm{AD}, \mathrm{JB}, \mathrm{H}, \mathrm{A}$ & $\mathrm{H}$ \\
\hline Rend. Em Excesso & $\mathrm{CS}, \mathrm{KS}, \mathrm{AD}, \mathrm{JB}, \mathrm{H}, \mathrm{A}$ & $\mathrm{AD}, \mathrm{H}, \mathrm{A}$ & $\mathrm{CS}, \mathrm{KS}, \mathrm{AD}, \mathrm{JB}, \mathrm{A}$ & $\mathrm{H}, \mathrm{A}$ & - & -.. & -- &.-- & -- \\
\hline \multicolumn{10}{|l|}{ Ponderado } \\
\hline Rend. Nominais & $\mathrm{CS}, \mathrm{KS}, \mathrm{AD}, \mathrm{H}, \mathrm{A}$ & $\mathrm{CS}, \mathrm{KS}, \mathrm{AD}, \mathrm{JB}, \mathrm{H}, \mathrm{A}$ & $\mathrm{CS}, \mathrm{KS}, \mathrm{AD}, \mathrm{H}, \mathrm{A}$ & $\mathrm{AD}, \mathrm{JB}, \mathrm{H}, \mathrm{A}$ & $\mathrm{KS}, \mathrm{AD}, \mathrm{H}, \mathrm{A}$ & A & ... & ... & ... \\
\hline Rend. Reais & $\mathrm{KS}, \mathrm{AD}, \mathrm{H}, \mathrm{A}$ & $\mathrm{CS}, \mathrm{KS}, \mathrm{AD}, \mathrm{JB}, \mathrm{A}$ & $\mathrm{CS}, \mathrm{KS}, \mathrm{AD}, \mathrm{H}, \mathrm{A}$ & $\mathrm{CS}, \mathrm{KS}, \mathrm{AD}, \mathrm{H}, \mathrm{A}$ & $\mathrm{CS}, \mathrm{KS}, \mathrm{AD}, \mathrm{JB}, \mathrm{H}, \mathrm{A}$ & $\mathrm{CS}, \mathrm{KS}, \mathrm{AD}, \mathrm{A}$ & -.. & -.. & -.- \\
\hline \multicolumn{10}{|l|}{ Não Ponderado } \\
\hline Rend. Nominais & $\mathrm{CS}, \mathrm{KS}, \mathrm{AD}, \mathrm{H}, \mathrm{A}$ & $\mathrm{CS}, \mathrm{KS}, \mathrm{AD}, \mathrm{JB}, \mathrm{H}, \mathrm{A}$ & $\mathrm{CS}, \mathrm{KS}, \mathrm{AD}, \mathrm{JB}, \mathrm{H}, \mathrm{A}$ & $\mathrm{CS}, \mathrm{KS}, \mathrm{AD}, \mathrm{JB}, \mathrm{A}$ & $\mathrm{CS}, \mathrm{KS}, \mathrm{AD}, \mathrm{H}, \mathrm{A}$ & A & -.. & -.. & -. \\
\hline Rend. Reais & $\mathrm{CS}, \mathrm{KS}, \mathrm{AD}, \mathrm{JB}, \mathrm{H}, \mathrm{A}$ & $\mathrm{CS}, \mathrm{KS}, \mathrm{AD}, \mathrm{JB}, \mathrm{H}, \mathrm{A}$ & $\mathrm{CS}, \mathrm{KS}, \mathrm{AD}, \mathrm{JB}, \mathrm{H}, \mathrm{A}$ & $\mathrm{CS}, \mathrm{KS}, \mathrm{AD}, \mathrm{JB}, \mathrm{A}$ & $\mathrm{KS}, \mathrm{AD}, \mathrm{JB}, \mathrm{H}, \mathrm{A}$ & A & $\cdots$ & -.- & -.- \\
\hline Rend. Em Excesso & $\mathrm{CS}, \mathrm{KS}, \mathrm{AD}, \mathrm{JB}, \mathrm{H}, \mathrm{A}$ & $\mathrm{CS}, \mathrm{KS}, \mathrm{AD}, \mathrm{JB}, \mathrm{H}, \mathrm{A}$ & $\mathrm{CS}, \mathrm{KS}, \mathrm{AD}, \mathrm{IB}, \mathrm{A}$ & $\mathrm{H}, \mathrm{A}$ & - & -.- & -.. & -.. & -.- \\
\hline \multicolumn{10}{|l|}{ Actividades Financeiras } \\
\hline Rend. Nominais & $\mathrm{CS}, \mathrm{KS}, \mathrm{AD}, \mathrm{JB}, \mathrm{H}, \mathrm{A}$ & A & $\mathrm{CS}, \mathrm{KS}, \mathrm{AD}, \mathrm{JB}, \mathrm{H}, \mathrm{A}$ & $\mathrm{CS}, \mathrm{H}, \mathrm{A}$ & - & -.. & ... & $\ldots$ & -.. \\
\hline Rend. Reais & $\mathrm{CS}, \mathrm{KS}, \mathrm{AD}, \mathrm{JB}, \mathrm{H}, \mathrm{A}$ & $\mathrm{H}, \mathrm{A}$ & $\mathrm{CS}, \mathrm{KS}, \mathrm{AD}, \mathrm{JB}, \mathrm{A}$ & $\mathrm{CS}, \mathrm{H}, \mathrm{A}$ & - & ... & $\ldots$ & $\ldots$ & -.. \\
\hline \multicolumn{10}{|c|}{ Indústrias Transformadoras } \\
\hline Rend. Nominais & $\mathrm{CS}, \mathrm{KS}, \mathrm{AD}, \mathrm{H}, \mathrm{A}$ & $\mathrm{CS}, \mathrm{KS}, \mathrm{AD}, \mathrm{JB}, \mathrm{A}$ & $\mathrm{KS}, \mathrm{AD}, \mathrm{H}, \mathrm{A}$ & $\mathrm{AD}, \mathrm{JB}, \mathrm{H}, \mathrm{A}$ & $\mathrm{H}, \mathrm{A}$ & $\mathrm{H}, \mathrm{A}$ & $\ldots$ & -.. & -.- \\
\hline Rend. Reais & $\mathrm{CS}, \mathrm{KS}, \mathrm{AD}, \mathrm{H}, \mathrm{A}$ & $\mathrm{CS}, \mathrm{KS}, \mathrm{AD}, \mathrm{JB}, \mathrm{A}$ & $\mathrm{A}$ & $\mathrm{CS}, \mathrm{KS}, \mathrm{AD}, \mathrm{H}, \mathrm{A}$ & $\mathrm{KS}, \mathrm{AD}, \mathrm{JB}, \mathrm{H}, \mathrm{A}$ & $\mathrm{AD}, \mathrm{A}$ & $\ldots$ & ... & ... \\
\hline Rend. Em Excesso & $\mathrm{CS}, \mathrm{KS}, \mathrm{AD}, \mathrm{JB}, \mathrm{H}, \mathrm{A}$ & $\mathrm{H}, \mathrm{A}$ & $\mathrm{CS}, \mathrm{KS}, \mathrm{AD}, \mathrm{JB}, \mathrm{A}$ & $\mathrm{KS}, \mathrm{A}$ & - & -.. & -.. & -.. & -.. \\
\hline \multicolumn{10}{|l|}{ Outras Indústrias } \\
\hline Rend. Nominais & $\mathrm{H}, \mathrm{A}$ & $\mathrm{CS}, \mathrm{KS}, \mathrm{AD}, \mathrm{JB}, \mathrm{H}, \mathrm{A}$ & $\mathrm{CS}, \mathrm{H}, \mathrm{A}$ & $\mathrm{JB}, \mathrm{A}$ & $\mathrm{KS}, \mathrm{AD}, \mathrm{JB}, \mathrm{H}, \mathrm{A}$ & . & $\ldots$ & $\ldots$ & ... \\
\hline Rend. Reais & $\mathrm{H}, \mathrm{A}$ & $\mathrm{CS}, \mathrm{KS}, \mathrm{AD}, \mathrm{JB}, \mathrm{A}$ & $\mathrm{CS}, \mathrm{AD}, \mathrm{H}, \mathrm{A}$ & $\mathrm{KS}, \mathrm{A}$ & $\mathrm{CS}, \mathrm{KS}, \mathrm{AD}, \mathrm{JB}, \mathrm{H}, \mathrm{A}$ & A & $\ldots$ & ..- & -.. \\
\hline Rend. Em Excesso & $\mathrm{AD}, \mathrm{JB}, \mathrm{H}, \mathrm{A}$ & $\mathrm{CS}, \mathrm{KS}, \mathrm{AD}, \mathrm{H}, \mathrm{A}$ & $\mathrm{CS}, \mathrm{KS}, \mathrm{AD}, \mathrm{JB}, \mathrm{A}$ & $\mathrm{H}, \mathrm{A}$ & - & -.. & $\cdots$ & -.- & -.- \\
\hline \multicolumn{10}{|l|}{$1^{\circ}$ Quartil } \\
\hline Rend. Nominais & $\mathrm{CS}, \mathrm{KS}, \mathrm{AD}, \mathrm{H}, \mathrm{A}$ & $\mathrm{CS}, \mathrm{KS}, \mathrm{AD}, \mathrm{JB}, \mathrm{H}, \mathrm{A}$ & $\mathrm{CS}, \mathrm{KS}, \mathrm{AD}, \mathrm{JB}, \mathrm{H}, \mathrm{A}$ & $\mathrm{AD}, \mathrm{JB}, \mathrm{H}, \mathrm{A}$ & $\mathrm{AD}, \mathrm{H}, \mathrm{A}$ & $\mathrm{H}, \mathrm{A}$ & $\ldots$ & $\ldots$ & -.. \\
\hline Rend. Reais & $\mathrm{CS}, \mathrm{KS}, \mathrm{AD}, \mathrm{JB}, \mathrm{H}, \mathrm{A}$ & $\mathrm{CS}, \mathrm{KS}, \mathrm{AD}, \mathrm{JB}, \mathrm{H}, \mathrm{A}$ & $\mathrm{CS}, \mathrm{KS}, \mathrm{AD}, \mathrm{H}, \mathrm{A}$ & $\mathrm{AD}, \mathrm{JB}, \mathrm{H}, \mathrm{A}$ & $\mathrm{CS}, \mathrm{AD}, \mathrm{H}, \mathrm{A}$ & A & -.. & ... & -.. \\
\hline Rend. Em Excesso & $\mathrm{CS}, \mathrm{KS}, \mathrm{AD}, \mathrm{JB}, \mathrm{H}, \mathrm{A}$ & A & $\mathrm{CS}, \mathrm{KS}, \mathrm{AD}, \mathrm{JB}, \mathrm{A}$ & $\mathrm{JB}, \mathrm{A}$ & $\mathrm{CS}, \mathrm{AD}$ & -.. & -.. & -.. & -.. \\
\hline \multicolumn{10}{|l|}{$3^{\circ}$ Quartil } \\
\hline \multicolumn{10}{|l|}{$4^{\circ}$ Quartil } \\
\hline Rend. Nominais & $\mathrm{CS}, \mathrm{KS}, \mathrm{AD}, \mathrm{JB}, \mathrm{H}, \mathrm{A}$ & $\mathrm{CS}, \mathrm{KS}, \mathrm{AD}, \mathrm{JB}, \mathrm{A}$ & $\mathrm{CS}, \mathrm{KS}, \mathrm{AD}, \mathrm{JB}, \mathrm{H}, \mathrm{A} C$ & $\mathrm{CS}, \mathrm{KS}, \mathrm{AD}, \mathrm{JB}, \mathrm{H}, \mathrm{A}$ & $\mathrm{CS}, \mathrm{H}, \mathrm{A}$ & A & ..- & -.. & -.. \\
\hline Rend. Reais & $\mathrm{CS}, \mathrm{KS}, \mathrm{AD}, \mathrm{JB}, \mathrm{H}, \mathrm{A}$ & $\mathrm{CS}, \mathrm{KS}, \mathrm{AD}, \mathrm{JB}, \mathrm{A}$ & $\mathrm{CS}, \mathrm{KS}, \mathrm{AD}, \mathrm{JB}, \mathrm{H}, \mathrm{A} C$ & $\mathrm{CS}, \mathrm{KS}, \mathrm{AD}, \mathrm{JB}, \mathrm{H}, \mathrm{A}$ & $\mathrm{JB}, \mathrm{A}$ & A & $\ldots$ & ... & -.. \\
\hline Rend. Em Excesso & $\mathrm{CS}, \mathrm{KS}, \mathrm{AD}, \mathrm{JB}, \mathrm{H}, \mathrm{A}$ & $\mathrm{CS}, \mathrm{KS}, \mathrm{AD}, \mathrm{JB}, \mathrm{H}, \mathrm{A}$ & $\mathrm{CS}, \mathrm{KS}, \mathrm{AD}, \mathrm{JB}, \mathrm{H}, \mathrm{A}$ & $\mathrm{H}, \mathrm{A}$ & $\mathrm{H}$ & -.- &.- & -.. & -.- \\
\hline
\end{tabular}

Nota: O traço (-) significa que não é possível rejeitar com um nível de confiança de $95 \%$ a hipótese de que os resíduos da regressão desse tipo de rendibilidades (nominais, reais ou em excesso a uma taxa de rendibilidade isenta de risco) desse índice e para esse período de investimento, segue uma distribuição normal, são homocedásticos e independentes. Os três traços (---) indicam que não foi possível, com os dados disponíveis, efectuar os testes de adequação para esse período de investimento. As iniciais $\mathrm{QQ}, \mathrm{KS}, \mathrm{AD}$ e JB correspondem aos testes de adequação utilizados, respectivamente o teste de Qui-Quadrado, o teste de Kolmogorov-Smirnov, o teste de Anderson-Darling e o teste de Jarque-Bera. Quando aparecer uma dessas iniciais, significa que é possível rejeitar a hipótese nula de que essa sucessão cronológica de resíduos segue uma distribuição normal, com um nível de significância de 5\%. As iniciais H e A correspondem aos testes de heterocedasticidade e autocorrelação utilizados, respectivamente o teste de White (1980) e o teste de multiplicador de Lagrange. Quando aparecer uma dessas iniciais, significa que é possível rejeitar a hipótese nula de que essa sucessão cronológica de resíduos é homocedástica ou independente, com nível de significância de 5\%.

Todos os resultados evidenciam o facto de que não é possível utilizar o teste tradicional de significância dos estimadores dos coeficientes da regressão, uma 
vez que estes não são estimadores eficientes. Uma forma de se utilizar o teste tradicional de significância dos estimadores é pelo recurso a matrizes de covariância consistentes na presença de heterocedasticidade e/ou autocorrelação, que irão corrigir o erro padrão dos estimadores, transformando-os em estimadores eficientes. Foram assim utilizados os procedimentos de correcção de heterocedasticidade de White (1980); o procedimento de correcção de heterocedasticidade e autocorrelação de Newey e West (1987); e o procedimento de correcção da autocorrelação gerada pela utilização de observações justapostas de Hansen e Hodrick (1980).

A Tabela 3, na seqüência, apresenta um resumo dos resultados dos testes paramétricos de significância do estimador do coeficiente beta da regressão para todos os índices analisados, todas as rendibilidades (nominais, reais e em excesso) e horizontes de investimento considerados.

A análise dos resultados dos testes paramétricos confirma que a presença de autocorrelação e heterocedasticidade leva a uma subestimação dos erros padrões dos estimadores da regressão, tornando assim possível a incorrecta rejeição da hipótese nula. Os resultados evidenciam a presença de comportamentos de reversão e/ou aversão à média dos índices de rendibilidade considerados. O procedimento de correcção que aparentemente proporciona os melhores resultados é o de Hansen e Hodrick (1980), o que não é estranho, se se tiver em consideração que é o único procedimento criado especificamente para corrigir o efeito de autocorrelação proporcionado pela utilização de observações justapostas. A generalidade dos resultados pode ser colocada em causa, uma vez que, como já foi referido, para muitos índices, rendibilidades e horizontes temporais considerados é possível rejeitar a hipótese de que os resíduos sigam uma distribuição normal.

Outra forma de testar a significância estatística dos resultados é a utilização de testes não-paramétricos, empregando, por exemplo, testes de hipóteses com recurso a computação intensiva. Estas metodologias de teste incluem os testes de amostragem de Monte Carlo, técnicas de alteração aleatória da ordem (com aproximação) ${ }^{(9)}$ e métodos de bootstrap resampling (para uma discussão sobre estes métodos ver Noreen, 1989).

O teste de hipóteses não-paramétrico considerado foi o teste de alteração aleatória da ordem, também utilizado por Kim, Nelson e Startz (1991), McQueen (1992) e Richardson (1993a). Esta metodologia permite testar a hipótese nula em que uma variável ou conjunto de variáveis não se relacionam com outra variável ou conjunto de variáveis. A alteração aleatória da ordem da série permite anular qualquer relação que possa existir entre as variáveis. Se as variáveis estiverem de alguma forma relacionadas, o valor da estatística do teste para os dados originais 
deve ser pouco usual, quando comparado com os valores da estatística após a ordem dos dados ter sido alterada aleatoriamente (Noreen, 1989).

\section{Tabela 3: Resumo dos Resultados dos Testes Paramétricos de Significância dos Estimadores do Coeficiente da Regressão pelo Método dos Mínimos Quadrados}

\begin{tabular}{|c|c|c|c|c|c|c|c|c|}
\hline \multirow[b]{2}{*}{ Índices } & \multirow{2}{*}{1} & \multicolumn{7}{|c|}{ Horizonte de investimento (em anos) } \\
\hline & & 2 & 3 & 4 & 5 & 6 & 7 & 8 \\
\hline \multicolumn{9}{|l|}{ BTA } \\
\hline Rend. Nominais & (A) $t, \mathrm{~W}$ & (A) $\mathrm{t}, \mathrm{W}$ & - & (R) $\mathrm{t}, \mathrm{W}$ & (R) $t, W, N W$ & (R) $t, W, N W$ & (R) $t, W, N W$ & (R) $t, W, N W$ \\
\hline Rend. Reias & (A) $t, W$ & . & (R) $t, \mathrm{~W}$ & (R) $t, W, N W$ & (R) t,W,NW & (R) $\mathrm{t}, \mathrm{W}, \mathrm{NW}$ & (R) $t, W, N W$ & (R) $\mathrm{t}, \mathrm{W}, \mathrm{NW}$ \\
\hline Rend. em Excesso & - & (A) t,W,NW & - & (A) $\mathrm{t}, \mathrm{W}, \mathrm{NW}$ & (A) $\mathrm{t}, \mathrm{W}, \mathrm{NW}$ & -- & $-\cdot$ & -.. \\
\hline \multicolumn{9}{|l|}{ Ponderado } \\
\hline Rend. Nominais & (A) $\mathrm{t}, \mathrm{W}$ & (A) $t, W, N W$ & (A) $t, W, N W$ & (A) $\mathrm{t}, \mathrm{W}, \mathrm{NW}$ & (A) $t, W, N W$ & (R) $\mathrm{t}, \mathrm{W}, \mathrm{NW}$ & ... & -.. \\
\hline Rend. Reias & (A) $\mathrm{t}, \mathrm{W}$ & (A) $t, W, N W$ & (A) $t, \mathrm{~W}, \mathrm{NW}$ & (A) $t, W, N W$ & (A) $\mathrm{t}, \mathrm{W}, \mathrm{NW}$ & (R) t,W & $\ldots$ & $\ldots$ \\
\hline Rend. em Excesso & . & (A) $t, W, N W$ & 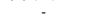 & (A) $\mathrm{t}, \mathrm{W}, \mathrm{NW}$ & (A) $t, W, N W$ & -.. & -.. & -.. \\
\hline \multicolumn{9}{|l|}{ Não Ponderado } \\
\hline Rend. Nominais & (A) $\mathrm{t}, \mathrm{W}$ & (A) $t, W, N W$ & (A) $t, \mathrm{~W}, \mathrm{NW}$ & (A) $\mathrm{t}, \mathrm{W}, \mathrm{NW}$ & (A) $\mathrm{t}, \mathrm{W}, \mathrm{NW}$ & (R) $\mathrm{t}, \mathrm{W}, \mathrm{NW}$ & ... & ... \\
\hline Rend. Reias & (A) $t, \mathrm{~W}$ & (A) $t, W, N W$ & (A) $t, \mathrm{~W}, \mathrm{NW}$ & (A) $t, \mathrm{~W}, \mathrm{NW}$ & (A) $\mathrm{t}, \mathrm{W}, \mathrm{NW}$ & (R) $t, W, N W$ & -.. & -.. \\
\hline Rend. em Excesso & (A) $\mathrm{t}$ & (A) $t, W, N W$ & (A) $t, \mathrm{~W}, \mathrm{NW}$ & (A) $t, \mathrm{~W}, \mathrm{NW}$ & (A) $t, \mathrm{~W}, \mathrm{NW}$ &.-- & --. & -.. \\
\hline \multicolumn{9}{|l|}{ Actividades Financeiras } \\
\hline Rend. Nominais & (A) $\mathrm{t}$ & (A) $t, \mathrm{~W}, \mathrm{NW}$ & (A) $t, \mathrm{~W}, \mathrm{NW}$ & (A) $\mathrm{t}, \mathrm{W}, \mathrm{NW}, \mathrm{HH}$ & (A) $t, W, N W, H H$ & -.- & -.- & -.. \\
\hline Rend. Reias & (A) $\mathrm{t}$ & (A) $t, W, N W$ & (A) $t, \mathrm{~W}, \mathrm{NW}$ & (A) $t, W, N W$ & (A) $t, W, N W, H H$ &.-- & -.- & -- \\
\hline Rend. em Excesso & (A) $\mathrm{t}$ & (A) $\mathrm{t}, \mathrm{W}, \mathrm{NW}$ & (A) $\mathrm{t}, \mathrm{W}, \mathrm{NW}$ & (A) $t, W, N W$ & (A) t,W,NW,HH & -- & -- & -- \\
\hline \multicolumn{9}{|l|}{ Comércio e Serviços } \\
\hline Rend. Nominais & (A) $\mathrm{t}, \mathrm{W}$ & (A) $t, W, N W$ & (A) $t, W, N W$ & (A) $\mathrm{t}, \mathrm{W}, \mathrm{NW}$ & (A) $t, W, N W$ & (R) $\mathrm{t}, \mathrm{W}, \mathrm{NW}$ & -- & -- \\
\hline Rend. Reias & (A) $\mathrm{t}, \mathrm{W}$ & (A) $t, W, N W$ & (A) t,W,NW & (A) $\mathrm{t}, \mathrm{W}, \mathrm{NW}$ & (A) $\mathrm{t}, \mathrm{W}, \mathrm{NW}$ & (R) $\mathrm{t}, \mathrm{W}, \mathrm{NW}$ & $\ldots$ & $\ldots$ \\
\hline Rend. em Excesso & - & (A) $\mathrm{t}, \mathrm{W}$ & (R) $\mathrm{t}$ & (A) $t, \mathrm{~W}$ & (A) $\mathrm{t}, \mathrm{W}, \mathrm{NW}$ & -.- & -.- & -.- \\
\hline \multicolumn{9}{|c|}{ Indústrias Transformadoras } \\
\hline Rend. Nominais & (A) $\mathrm{t}, \mathrm{W}$ & (A) $\mathrm{t}, \mathrm{W}$ & (A) $t, \mathrm{~W}, \mathrm{NW}$ & (A) $t, \mathrm{~W}, \mathrm{NW}$ & (A) t,W,NW & - & ... & ... \\
\hline Rend. Reias & (A) $t, \mathrm{~W}$ & 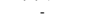 & (A) $\mathrm{t}, \mathrm{W}$ & (A) $t, \mathrm{~W}$ & (A) $t, W, N W$ & (A) $\mathrm{t}$ & -.. & -.- \\
\hline Rend. em Excesso & - & - & (A) $\mathrm{t}, \mathrm{W}$ & (A) $t, W, N W$ & - & $\ldots$ & -.- & -- \\
\hline \multicolumn{9}{|l|}{ Outras Indústrias } \\
\hline Rend. Nominais & (A) $t, W$ & (A) $t, \mathrm{~W}, \mathrm{NW}$ & (A) $t, \mathrm{~W}, \mathrm{NW}$ & (A) $t, W, N W$ & (A) $t, W, N W$ & (R) $t, \mathrm{~W}, \mathrm{NW}$ & -.. & -.. \\
\hline Rend. Reias & (A) $\mathrm{t}, \mathrm{W}$ & (A) $t, W, N W$ & (A) $t, \mathrm{~W}, \mathrm{NW}$ & (A) $t, W, N W$ & (A) $\mathrm{t}, \mathrm{W}$ & (R) $\mathrm{t}, \mathrm{W}, \mathrm{NW}$ & -- & -- \\
\hline Rend. em Excesso & - & (A) $\mathrm{t}, \mathrm{W}$ & (R) $t, W$ & (A) $\mathrm{t}, \mathrm{W}$ & - & -.- & -- & -.. \\
\hline \multicolumn{9}{|l|}{$1^{\circ}$ Quartil } \\
\hline Rend. Nominais & (A) $\mathrm{t}$ & (A) $t, \mathrm{~W}, \mathrm{NW}$ & (A) $\mathrm{t}, \mathrm{W}, \mathrm{NW}$ & (A) $\mathrm{t}, \mathrm{W}, \mathrm{NW}, \mathrm{HH}$ & (A) $t, W, N W$ & (A) $\mathrm{t}, \mathrm{W}$ & -.. & -.. \\
\hline Rend. Reias & - & (A) $t, W, N W$ & (A) $t, \mathrm{~W}, \mathrm{NW}$ & (A) $t, W, N W$ & (A) $t, W, N W$ & (R) $t, W, N W$ & -.. & -.- \\
\hline Rend. em Excesso & (R) $\mathrm{t}$ & (A) $\mathrm{t}, \mathrm{W}$ & (A) $t, \mathrm{~W}$ & (A) $t, W, N W$ & (A) $t, W, N W$ & $--\cdot$ & -- & -.- \\
\hline \multicolumn{9}{|l|}{$2^{\circ}$ Quartil } \\
\hline Rend. Nominais & (A) $t, W$ & (A) $\mathrm{t}, \mathrm{W}$ & (A) $t, \mathrm{~W}, \mathrm{NW}$ & (A) $t, \mathrm{~W}$ & (A) $t, W, N W$ & - & ... & -.. \\
\hline Rend. Reias & (A) $t, W$ & (A) $\mathrm{W}$ & (A) $\mathrm{t}, \mathrm{W}$ & - & (A) $\mathrm{W}$ & (A) $\mathrm{W}$ & -- & -.. \\
\hline Rend. em Excesso & 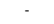 & (A) $\mathrm{t}, \mathrm{W}$ & (R) $\mathrm{t}, \mathrm{W}$ & - & (A) t,W,NW & -- & $\cdots$ & -- \\
\hline \multicolumn{9}{|l|}{$3^{\circ}$ Quartil } \\
\hline Rend. Nominais & (A) $t, W$ & (A) $t, \mathrm{~W}, \mathrm{NW}$ & (A) $t, W, N W$ & (A) t,W,NW,HH & (A) $t, W, N W$ & - & -.- & $\ldots$ \\
\hline Rend. Reias & (A) $\mathrm{t}, \mathrm{W}$ & (A) $t, \mathrm{~W}, \mathrm{NW}$ & (A) $t, \mathrm{~W}, \mathrm{NW}$ & (A) $\mathrm{t}, \mathrm{W}, \mathrm{NW}, \mathrm{HH}$ & (A) $\mathrm{t}, \mathrm{W}, \mathrm{NW}$ & (R) $\mathrm{t}, \mathrm{W}, \mathrm{NW}$ & -- & -- \\
\hline Rend. em Excesso & (A) $\mathrm{t}, \mathrm{W}$ & (A) $t, \mathrm{~W}, \mathrm{NW}$ & (A) $t, \mathrm{~W}, \mathrm{NW}$ & (A) $\mathrm{t}, \mathrm{W}, \mathrm{NW}$ & - & - & -- & -- \\
\hline \multicolumn{9}{|l|}{$4^{\circ}$ Quartil } \\
\hline Rend. Nominais & (A) $\mathrm{t}, \mathrm{W}$ & (A) $t, W, N W$ & (A) $t, W, N W$ & (A) $\mathrm{t}, \mathrm{W}, \mathrm{NW}, \mathrm{HH}$ & (A) t,W,NW & (A) $\mathrm{t}, \mathrm{W}, \mathrm{NW}$ & -.. & -.. \\
\hline Rend. Reias & (A) $\mathrm{t}$ & (A) $\mathrm{t}, \mathrm{W}$ & (A) $t, W, N W$ & (A) $t, W, N W$ & (A) $t, W, N W$ & (A) $t, W, N W$ & ... & -.. \\
\hline Rend. em Excesso & : & (A) $t, \mathrm{~W}, \mathrm{NW}$ & . & (A) $t, \mathrm{~W}, \mathrm{NW}$ & (A) $t, W, N W$ & $\ldots$ & $\ldots$ & $\ldots$ \\
\hline
\end{tabular}

Nota: O traço (-) significa que não é possível rejeitar, com nível de confiança de 95\%, a hipótese de que essa sucessão cronológica desse tipo de rendibilidades (nominais, reais ou em excesso a uma taxa de rendibilidade isenta de risco) desse índice e para esse período de investimento, siga um percurso aleatório. Os três traços (---) indicam que não foi possível, com os dados disponíveis, efectuar esses testes para esse período de investimento. As iniciais t, W, NW e HH correspondem, respectivamente, ao teste de $t$, ao teste de $\mathrm{t}$ com a variância do estimador $\beta$ corrigido da heterocedasticidade de acordo com White (1980), ao teste de $t$ com a variância do estimador $\beta$ corrigido da autocorrelação e heterocedasticidade de acordo com Newey e West (1987) e ao teste de $t$ com a variância do estimador $\beta$ corrigido da autocorrelação de acordo com Hansen e Hodrick (1980). Quando aparecer uma dessas iniciais, significa que é possível rejeitar a hipótese nula de que essa sucessão cronológica de rendibilidades siga percurso aleatório, com nível de significância de $5 \%$. 
A utilização desta metodologia de teste dos estimadores dos coeficientes de regressão apresenta a vantagem de não termos de assumir o pressuposto de normalidade dos resíduos da regressão, que não tem aderência no mercado accionista português, nem termos de basear as nossas conclusões em estatísticas baseadas em correcções que apenas têm validade assimptótica ${ }^{(10)}$. Existe ainda a vantagem de que esta metodologia permite a obtenção de resultados tão válidos como os testes paramétricos tradicionais, mesmo quando os pressupostos desses testes são válidos (Noreen, 1989). Para além da normalidade, estes testes são ainda eficientes, mesmo na presença de heterocedasticidade incondicional e autocorrelação, uma vez que a utilização do método de regressão pelos mínimos quadrados, que desconsidere estes problemas, não afecta a consistência dos seus estimadores, porque influencia apenas a sua eficiência ${ }^{(11)}$. Ora, como se está a utilizar um método não-paramétrico, isto deixa de constituir qualquer problema.

O teste de significância dos estimadores dos coeficientes da regressão, pelo recurso ao procedimento de alteração aleatória da ordem, pode ser dividido em três passos/fases. No primeiro passo, calcula-se o estimador dos coeficientes da regressão para os dados originais. O segundo passo consiste num ciclo de $n$ iterações. Em cada iteração desse ciclo, a ordem da série das rendibilidades mensais será alterada aleatoriamente, assegurando assim que não existe nenhuma dependência cronológica nessa sucessão de rendibilidades. As rendibilidades de médio e longo prazo são depois calculadas com base nas rendibilidades mensais, calculando-se de seguida o estimador dos coeficientes da regressão ${ }^{(12)}$. O terceiro passo, inicia-se no final de todas as iterações, e consiste no cálculo do seguinte quociente (Noreen, 1989):

$$
n s=\frac{n m i+1}{n i+1}
$$

em que:

$n s$ - nível de significância estatística do teste;

$n i$ - número de iterações;

$n m i$ - número de vezes em que o valor do beta calculado com base em valores das variáveis, cuja ordem foi alterada aleatoriamente, é menor ou igual ao valor de beta para os dados originais.

Rejeita-se a hipótese nula, se $n s$ for superior ao nível de significância do teste $(\alpha)$.

Para garantir a validade dos resultados tanto da simulação utilizada para esti- 
mar o enviesamento dos estimadores dos coeficientes da regressão, como do teste de alteração aleatória da ordem, foi necessário assegurar que o algoritmo de geração de números pseudo-aleatórios utilizado passava os testes de independência e de adequação a uma distribuição de probabilidade uniforme $[0,1]^{(13)}$. Foram testados três dos algoritmos sugeridos por Noreen (1989), por Press et al. (1986) e a função RND() da folha de cálculo Microsoft Excel v.7.0. Os testes utilizados foram sugeridos por Siegel (1956); Conover (1980); e por Law e Kelton (1991).

Para testar a independência dos números pseudo-aleatórios foram utilizadas três variantes do teste de sequências, e coeficientes de autocorrelação para vários desfasamentos. Para se testar formalmente a hipótese de adequação desses números pseudo-aleatórios a uma distribuição uniforme [0,1], utilizaram-se os testes de adequação de Qui-Quadrado para uma, duas e três dimensões; o teste de Kolmogorov-Smirnov; e o teste de Anderson-Darling. Todos os testes foram utilizados para testar uma sucessão de 32.768 números pseudo-aleatórios e para testar 10.000 sucessões de 235 números pseudo-aleatórios.

O algoritmo escolhido foi o designado de RAN1, sugerido por Noreen (1989) e por Press et al. (1986). Este algoritmo passou em todos os testes a que foi sujeito, assegurando assim a validade dos resultados obtidos pela simulação e pelo teste de alteração aleatória da ordem.

Os resultados dos testes não-paramétricos de significância dos estimadores dos coeficientes da regressão são apresentados na Tabela 4. O $\beta$ corrigido de enviesamento representa o estimador do coeficiente da regressão corrigido do enviesamento provocado pela utilização de um número relativamente reduzido de observações, e observações justapostas, para o cálculo das rendibilidades de médio e longo prazo. O enviesamento foi estimado com recurso à simulação, como já foi descrito. $\mathrm{O}$ valor da probabilidade mede a probabilidade de se obter um coeficiente da regressão com valor igual ao $\beta$ original, ou menor, corrigido de enviesamento e assumindo a hipótese de independência das rendibilidades. Valores da probabilidade inferiores a $5 \%$ indicam a presença de reversão para a média, ao passo que valores da probabilidade superiores a $95 \%$ indicam a presença de aversão à média.

Os resultados demonstram que a evidência de reversão e aversão à média anteriormente detectada através de testes paramétricos pode ser atribuída à violação de um ou mais pressupostos da regressão pelo método dos mínimos quadrados. Os procedimentos de correcção utilizados nos testes paramétricos têm validade assimptótica, o que pode explicar a pouca eficácia dessa correcção, uma vez que estamos a trabalhar com séries relativamente reduzidas. 


\section{Tabela 4: Resumo dos Resultados dos Testes Não-Paramétricos de Significância dos Estimadores do Coeficiente da Regressão pelo Método dos Mínimos Quadrados}

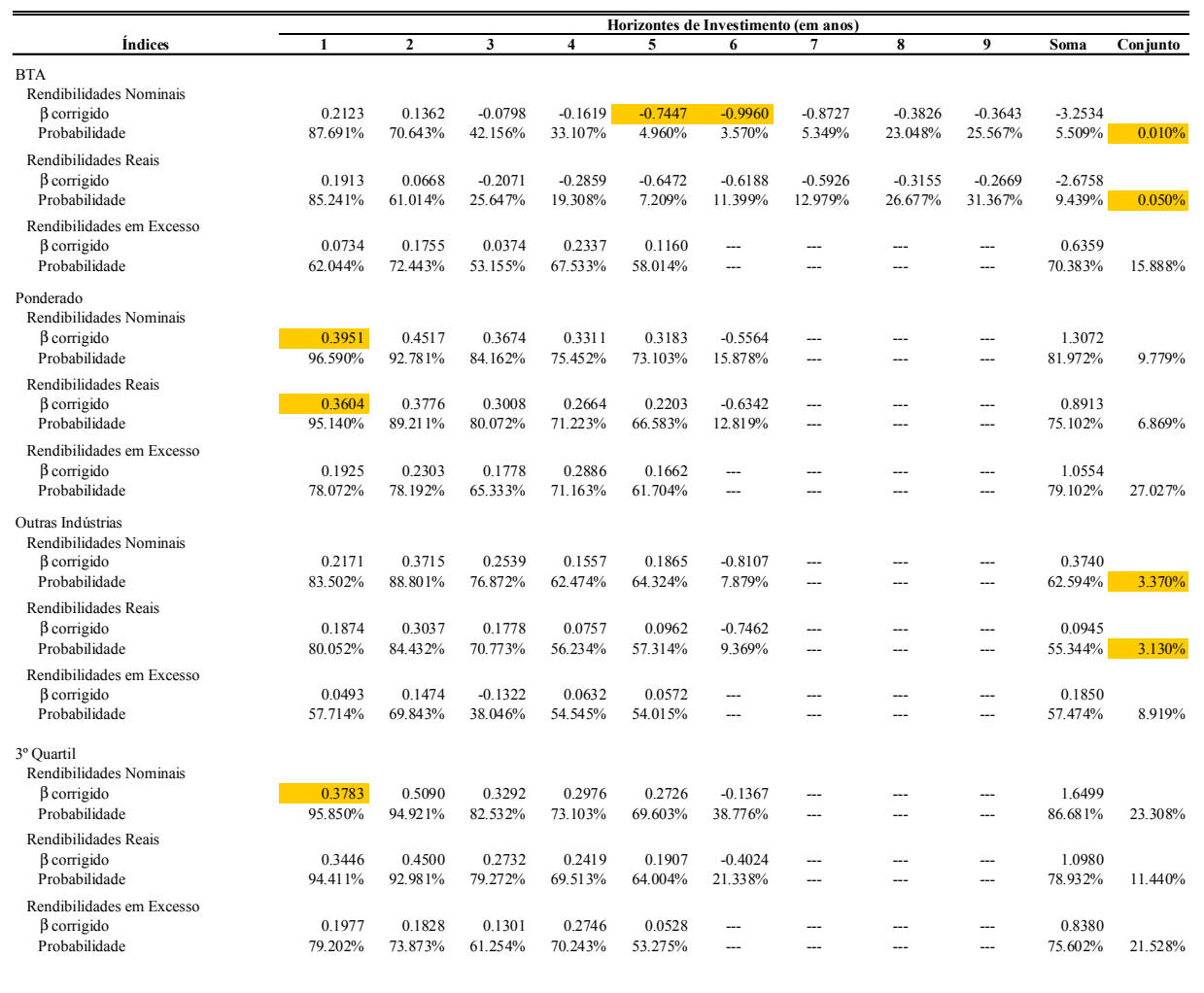

Nota: Apenas se apresentam os índices que comportam algum resultado estatisticamente significativo. $O$ valor da probabilidade dá-nos a probabilidade de obter-se um valor do coeficiente da regressão $(\beta)$ igual ou inferior ao valor obtido para a sucessão de rendibilidades considerada, no caso dessa sucessão cronológica de rendibilidade seguir um percurso aleatório. A significância estatística dos $\beta$ da regressão foi obtida através da utilização da técnica de alteração aleatória da ordem com 10.000 iterações. O valor do $\beta$ corrigido já foi expurgado do enviesamento provocado pela utilização de observações justapostas para o cálculo das rendibilidades de médio e longo prazo e pela utilização de amostras de reduzida dimensão. Um valor de $\beta$ igual a 0 indica que essa sucessão segue percurso aleatório; se for superior a zero, revela a presença de aversão à média; um valor inferior a 0 , manifesta a presença do fenómeno de reversão para a média das rendibilidades dos índices de acções. As duas últimas colunas apresentam os resultados dos testes de significância conjunta dos $\beta$ para todos os horizontes de investimento. A coluna correspondente à soma apresenta um teste conjunto, que se baseia na probabilidade de se obter um valor do somatório de todos os $\beta$ igual ou menor ao valor proporcionado pela amostra, assumindo que essa sucessão cronológica de rendibilidade segue um percurso aleatório. A última coluna, designada de conjunto, apresenta apenas o valor da probabilidade de se obter simultaneamente $\beta$ para todos os horizontes de investimento considerados, iguais ou tão baixos como os proporcionados pela amostra. Os valores sombreados representam valores estatisticamente significativos, com grau de confiança de $95 \%$. 
Quando se utiliza a técnica de alteração aleatória da ordem, encontra-se evidência de reversão para a média apenas para rendibilidades nominais do índice BTA, para horizontes de investimento de 5 e 6 anos. A evidência de aversão à média foi também ela significativamente reduzida, uma vez que agora só é possível encontrar resultados estatisticamente significativos para rendibilidades nominais e reais de um horizonte de investimento de 1 ano do índice não ponderado, e para rendibilidades nominais de um horizonte de investimento de 1 ano do índice do $3^{\circ}$ quartil.

Tal como Richardson (1993b) faz referência, a evidência de aversão e reversão para a média pode ser incorrectamente interpretada, se for dada muita importância aos resultados individuais de cada um dos horizontes de investimento. Para se ter em conta as possíveis interdependências dos resultados da regressão, ao longo de vários horizontes de investimento, foram utilizados dois testes conjuntos de significância estatística. O primeiro, designado de Soma, foi sugerido por McQueen (1992), e pode ser utilizado da seguinte forma: em primeiro lugar soma-se os coeficientes beta da regressão para todos os horizontes de investimento considerados; em seguida e utilizando o procedimento de alteração aleatória da ordem da série, determina-se a probabilidade de se obter um valor menor ou igual ao obtido no passo anterior, se as rendibilidades seguissem um percurso aleatório.

Em nossa opinião, este teste pode proporcionar conclusões incorrectas: quando se somam os estimadores betas das regressões para vários horizontes de investimento, os valores dos estimadores betas de menor valor serão compensados por estimadores beta de maior valor. Assim, propomos um teste que utiliza o procedimento de alteração aleatória da ordem, que designamos de Conjunto: ele testa formalmente a hipótese de se obter simultaneamente, para cada um dos horizontes de investimento considerados, um estimador do coeficiente beta da regressão menor ou igual ao valor obtido para os dados originais, se a sucessão de rendibilidades for independente.

Como é possível observar pela análise dos resultados dos testes conjuntos, a significância estatística do teste Conjunto é menor que a do teste Soma. Apenas para rendibilidades nominais e reais do índice BTA e do índice Outras Indústrias, é possível rejeitar a hipótese de que essas rendibilidades seguem percurso aleatório.

\section{CONCLUSÓES}

Tal como noutros estudos internacionais, podemos concluir que a evidência de 
reversão para a média e aversão à média é influenciada pelos tipos de testes de significância utilizados.

Os resultados do estudo são mistos, uma vez que encontramos evidência da presença de reversão para a média e de aversão à média das rendibilidades de índices de acções do mercado português. Os resultados sugerem ainda que a evidência é mais significativa para rendibilidades nominais e reais. Não foi encontrado nenhum tipo de dependência das rendibilidades em excesso. Isto pode ser explicado, em nossa opinião, pela diferença dos períodos de análise das rendibilidades em excesso em relação às rendibilidades nominais e reais, ou pela existência de uma relação entre a dependência cronológica das rendibilidades de médio e longo prazo e alguma variável macro-económica, que possa explicar essa forma de dependência sem rejeitar a hipótese de as rendibilidades seguirem percurso aleatório.

A evidência de reversão e aversão à média pode ter sido causada pelo período de euforia que existiu no mercado de capitais português, e que antecedeu o desaire bolsista de 1987. Os resultados podem ainda ser explicados pela existência de prémios de risco variáveis.

A evidência de aversão à média pode ser explicada pela infrequência de transacções, que caracterizou o mercado accionista português nos primeiros anos do período analisado. Alternativamente, pode ainda ser explicada pelo relativamente elevado ritmo de crescimento económico que se tem verificado em Portugal nos últimos anos.

Em conclusão, os resultados obtidos não são, em nossa opinião, suficientemente fortes para colocar em causa a eficiência do mercado accionista português, sem que antes se proceda a mais estudos empíricos, estudos estes que passarão necessariamente pelo alargamento do período de análise (pelo que teremos ainda de aguardar mais algum tempo), pela análise de outras variáveis que possam explicar esta autocorrelação, sem colocar em causa a eficiência dos mercados, ou até pelo teste de estratégias de comercialização de acções que tenham em consideração os resultados obtidos pelo nosso estudo, permitindo assim verificar se essas estratégias seriam capazes de gerar, sistematicamente, rendibilidades ajustadas ao risco, superiores a uma estratégia de aquisição e manutenção de um índice.

\section{NOTAS}

${ }^{1}$ Lee, Shleifer e Thaler (1990, p. 153) definem anomalia do seguinte modo: "Um resultado empírico qualifica-se como anómalo se é difícil de o «racionalizar», ou se é necessário assu- 
mir pressupostos implausíveis para explicar de acordo com o paradigma".

${ }^{2}$ Para uma demonstração ver Fama e French (1988), Forbes (1996) ou Areal (1997).

${ }^{3}$ Não se pretende de forma alguma fazer uma revisão exaustiva de todos os artigos publicados sobre o tema; limitamo-nos a apresentar aqueles que julgamos serem os mais relevantes.

${ }^{4} \mathrm{O}$ reduzido número de observações mensais destes índices impediu a sua inclusão neste trabalho.

${ }^{5}$ Os índices por quartis só foram calculados a partir de 1 de março de 1983, e o índice do sector de actividades financeiras a partir de 9 de outubro de 1985.

${ }^{6}$ A carteira do índice apresenta uma representatividade que oscila entre os $55 \%$ aos $90 \%$ da carteira de mercado representada pelo índice geral de acções da Bolsa de Valores de Lisboa.

${ }^{7}$ A razão de ser da escolha da taxa média ponderada da última colocação, no mercado primário anterior a cada mês, decorre da maior probabilidade de serem esses os bilhetes de Tesouro disponíveis para investir nesse mês.

${ }^{8}$ Os resultados detalhados destes e de todos os restantes testes que a seguir se apresentam estão disponíveis mediante solicitação aos autores.

${ }^{9}$ Designados na literatura anglo-saxónica de approximate randomization tests ou permutation tests.

${ }^{10}$ Esta é a grande vantagem desta metodologia, uma vez que estamos a trabalhar com amostras de reduzida dimensão.

${ }^{11}$ Ver, por exemplo, Greene (1993).

${ }^{12}$ Todos os testes de alteração aleatória da ordem foram executados com recurso a 10.000 iterações.

${ }^{13}$ Utiliza-se a designação de pseudo-aleatório ao invés da designação aleatório, uma vez que em rigor estes números são gerados através de um algoritmo, não sendo assim, por definição, aleatórios.

\section{REFERENCIAS BibLIOGRÁFICAS}

AREAL, N. M. DE P. B. DA C.

Reversão e aversão para a média da rendibilidade de índices de acções no mercado accionista português. Braga, 1997. Dissertação (Mestrado) - Escola de Economia e Gestão, Universidade do Minho, Portugal.
ASSOCIAÇÃO DA BOLSA DE VALORES DO PORTO.

Futuros sobre o índice PSI-20. Porto, Portugal, 1995.

BANZ, R. W.

The relationship between returns and market value of common stocks. Journal of Financial Economics, v. 9, p. 03-18, 1981. 
CONOVER, W. J.

Practical nonparametric statistics. 2. ed. New York : John Wiley \& Sons, 1980.

COSTA, C. S. T.;

SOUSA, P. M. M.

Construção de índices de remuneração da bolsa de valores de Lisboa. Working paper, Universidade de Lisboa, 1988.

FAMA, E. F.

Efficient capital markets : a review of theory and empirical work. Journal of Finance, v. 25, n. 2, p. $383-417,1970$.

Foundations of finance. New York : Basic Books, 1976.

Efficient capital market, II. Journal of Finance, v. 46, n. 5, p. $1575-1618,1991$.

FAMA, E. F.;

FRENCH, K. R.

Permanent and temporary components of stock prices. Journal of Political Economy, v. 96, n. 2, p. 246-273, 1988.

FONSECA, F. A.;

CONFRARIA, J.;

PINHEIRO, J.

Metodologia dos índices oficiais de valores mobiliários de rendimento variável. Lisboa : Bolsa de Valores de Lisboa/Universidade Católica Portuguesa, 1990.
FORBES, W. P.

Picking winners? A survey of the mean reversion and overreaction of stock prices literature. Journal of Economic Surveys, v. 10, n. 2, p. 123-136, 1996.

GREENE, W. H.

Econometric analysis. 2. ed. Englewood Cliffs, NJ : PrenticeHall, 1993.

HANSEN, L. P.;

HODRICK, R. J.

Forward exchange rates as optimal predictors of future spot rates : an econometric analysis. Journal of Political Economy, v. 88 , n. 5, p. 829-853, 1980.

JEGADEESH, N. (1990)

Evidence of predictable behaviour of security returns. Journal of Finance, v. 45, n. 3, p. 881-898, 1990.

KENDALL, M. G.

Note on bias in the estimation of autocorrelation. Biometrika, v. 41, n. 3-4, p. 403-404, 1954.

KIM, M. J.;

NELSON, C. R.;

STARTZ, R.

Mean reversion in stock prices? A reappraisal of the empirical evidence. Review of Economic Studies, v. 58, n. 195, p. 515-528, 1991. 
KING, B. F.

Market and industry factors in stock price behavior. Journal of Business, v. 39, p. 139-190, 1966.

LAW, A. M.;

KELTON, W. D.

Simulation modeling and analysis. 2. ed. New York : McGraw-Hill, 1991.

LEE, C. M. C.;

SHLEIFER, A.;

THALER, R. H.

Anomalies : closed-end mutual funds. Journal of Economic Perspectives, v. 4, n. 4, p. 153164, Fall 1990.

MARRIOTT, F. H. C.;

POPE, J. A.

Bias in the estimation of autocorrelation. Biometrika, v. 41, n. 3-4, p. 390-402, 1954.

MCQUEEN, G.

Long-horizon mean-reverting stock prices revisited. Journal of Financial and Quantitative Analysis, v. 27, n. 1, p. 01-18, 1992.

MCQUEEN, G.;

THORLEY, S.

Are stock returns predictable? A test using markov chains. Journal of Finance, v. 46, n. 1, p. 239264, 1991.
NEWEY, W. K.;

WEST, $\mathrm{K}$.

A simple, positive semi-definite, heteroskedasticity and autocorrelation consistent covariance matrix. Econometrica, v. 55, n. 3, p. 703708, 1987.

NOREEN, E. W.

Computer intensive methods for testing hypothesis : an introduction. New York : John Wiley \& Sons, 1989.

POTERBA, J. M.;

SUMMERS, L. H.

Mean reversion in stock prices : evidence and implications. Journal of Financial Economics, v. 22, n. 1, p. 27-59, 1988.

PRESS, W. H. et al.

Numerical recipes - the art of scientific computing. Cambridge : Cambridge University Press, 1986.

RICHARDSON, G. M.

Mean reversion : market anomaly or empirical misnomer? Arlington, 1993a. Doctoral Thesis - University of Texas.

Temporary components of stock prices : a skeptic's view. Journal of Business and Economic Statistics, v. 11, n. 2, p. 199-207, 1993b. 
RICHARDSON, M.;

STOCK, J. H.

Drawing inferences from statistics based on multiyear asset returns. Journal of Financial Economics, v. 25, n. 2, p. 323-348, 1989.

SIEGEL, S.

Nonparametric statistics for the behavioral sciences. New York : McGraw-Hill, 1956.
SUMMERS, L. H.

Does the stock market rationally reflect fundamental values? Journal of Finance, v. 41, n. 3, p. 591-601, 1986.

WHITE, H.

A heteroskedasticity-consistent covariance matrix estimator and a direct test for heteroskedasticity. Econometrica, v. 48, n. 4, p. 817838, 1980. 